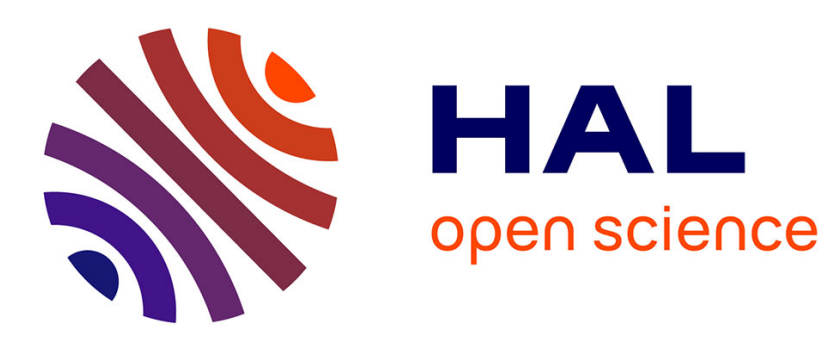

\title{
Simulation globale en réseau pour le FLE ? La plate-forme informatique MEPA-2D
}

Jérôme Lehuen, Sylwia Kitlinska

\section{To cite this version:}

Jérôme Lehuen, Sylwia Kitlinska. Simulation globale en réseau pour le FLE ? La plate-forme informatique MEPA-2D. STICEF (Sciences et Technologies de l'Information et de la Communication pour l'Éducation et la Formation), 2006, 13, 23 p. hal-00696334

\section{HAL Id: hal-00696334 \\ https://hal.science/hal-00696334}

Submitted on 11 May 2012

HAL is a multi-disciplinary open access archive for the deposit and dissemination of scientific research documents, whether they are published or not. The documents may come from teaching and research institutions in France or abroad, or from public or private research centers.
L'archive ouverte pluridisciplinaire HAL, est destinée au dépôt et à la diffusion de documents scientifiques de niveau recherche, publiés ou non, émanant des établissements d'enseignement et de recherche français ou étrangers, des laboratoires publics ou privés. 


\title{
Simulation Globale en Réseau pour le FLE ? La Plate-forme Informatique MEPA-2D
}

\author{
Jérôme LEHUEN, Sylwia KITLINSKA [LIUM, Le Mans]
}

- RÉSUMÉ : Cet article traite d'environnements de type "jeux de rôles en réseau" pour l'apprentissage d'une langue étrangère. A partir d'un bref état de l'art, nous dégageons un ensemble de critères qui permettent de caractériser les plates-formes dédiées à la pratique communicative d'une langue étrangère, ou utilisées comme telles dans le cadre d'activités de type jeux de rôles. Ensuite, nous présentons MEPA2D, une nouvelle plate-forme que nous avons prototypée et mise à l'essai dans des contextes et avec des publics variés. MEPA-2D est un système client-serveur qui permet à des apprenants, à des enseignants, et à des agents logiciels d'agir et d'interagir à distance dans un monde virtuel partagé. En favorisant la collaboration au sein d'un groupe d'apprenants encadrés par un réseau de tuteurs, MEPA-2D tente d'articuler les principes du socioconstructivisme, de l'étayage Brunerien et de l'apprentissage collaboratif.

nOTS CLÉS : Apprentissage d'une Langue, Simulation, Communication, Jeux de Rôles, FLE

- ABSTRACT : This article deals with Role Playing Game (RPG) environments for language learning. From a short state-of-the-art, a set of criteria is put forward in order to specify environments to support the communicative practice of a foreign language in the context of RPG activities. Then, MEPA-2D is described. It is a new RPG platform dedicated to foreign language training. It is based on a server system infrastructure which allows learners, teachers, and software agents to act and to interact remotely in a shared virtual world. Eventually, the first test campaign is presented. The latter has been carried out in several contexts and with a varied public. Then, some research perspectives are put forward.

KEYWORDS : CALL, Simulation, Communication, Role Playing Games, French as a Foreign Language

1. Introduction

2. La simulation globale

3. Quatre environnements

4. La plate-forme MEPA-2D

5. Scénarisation pour MEPA-2D

6. Mises à l'épreuve du dispositif

7. Conclusion

BIBLIOGRAPHIE

SITOGRAPHIE (derniers accès le 06/10/06)

\section{Introduction}

L'approche communicative décrite par la didactique des langues étrangères vise à développer des compétences de communication langagière dans un contexte socioculturel donné. Le postulat psychologique qui sous-tend cette approche est qu'apprendre une langue, c'est s'entraîner dès le départ à penser et à parler en langue étrangère (Puren, 2001), les apprentissages émergent dans et par 
l'interaction. Le Cadre Commun Européen de Référence (Conseil de l'Europe, 2001) affine l'idée en présentant l'approche communicative dans une perspective actionnelle, centrée sur la relation entre les stratégies de l'acteur et la ou les tâche(s) à réaliser dans un environnement et dans des conditions données. Les stratégies se matérialisent alors sous la forme des actions (langagières ou non suivant la nature de la tâche) que produit l'apprenant dans l'accomplissement d'une tâche donnée.

Les exercices de référence de l'approche communicative/actionnelle sont les simulations et les jeux de rôle. En abordant les thèmes anthropologiques de la civilisation cible, ces activités ont pour but de créer une mise en situation suffisante afin de contextualiser la parole et rendre ainsi les enjeux communicatifs plus authentiques. Or, cette authenticité semble particulièrement difficile à retrouver notamment dans le milieu hétéroglotte ${ }^{(1)}$. Tout d'abord, contrairement aux milieux hétérogènes, le recours à la langue cible apparaît fortement conventionnel entre apprenants d'une même L1 (langue maternelle). Ensuite, une homogénéité culturelle et une faible expérience de la "socioculture" favorisent chez les apprenants la fossilisation des représentations simplifiées (ethnocentrisme). Toutefois, de multiples expériences interculturelles prouvent que la confrontation avec des représentations stéréotypées de la "socioculture" cible venant d'autres horizons mène souvent les apprenants à réviser les leurs et à y porter un regard plus attentif (De Carlo, 1998). Il en découle qu'un contact plus large avec la société cible et/ou une ouverture vers d'autres milieux hétéroglottes peuvent apporter certains avantages. Ainsi, à l'ère du numérique, il semble intéressant d'examiner le potentiel des technologies actuelles pour la création d'une telle ouverture, par exemple sous la forme de "classes virtuelles hétérogènes" (L1 et culture) et fonctionnant dans des contextes cibles simulés.

En insistant sur la communication-expression en langue et sur la reconstitution aussi fidèle que possible du réel (Debyser, 1974), la pratique de simulation globale devient notre pratique de référence. Dans la suite de cet article, nous examinerons ses caractéristiques pour ne retenir que celles qui semblent particulièrement intéressantes à préserver dans une tentative de transposition informatique. Ensuite, nous passerons en revue quatre environnements dédiés à l'apprentissage des langues qui prétendent s'inscrire dans le cadre théorique défini par l'approche communicative et/ou qui permettent de mettre en place des activités qui s'apparentent à de la simulation globale. Le but de cette revue est d'observer dans quelle mesure et avec quel résultat il est possible de transposer certains de ses éléments pour son adaptation en réseau. Enfin, nous présenterons la plate-forme informatique MEPA-2D qui tente de s'inscrire dans un cadre théorique, didactique, et technologique que nous préciserons.

\section{La simulation globale}

\subsection{Définition}

Une simulation globale (SG) est une sorte de jeu de rôles "en vraie grandeur" dans lequel des apprenants doivent agir et interagir en endossant une identité fictive (Debyser, 1991), (Yaiche, 1996). Une SG fonctionne selon deux principes fondamentaux : un lieu-thème qui permet de convoquer et de fédérer les activités pédagogiques traditionnellement atomisées, et des identités fictives qui permettent aux participants de se glisser dans la peau d'un personnage. L'intérêt d'une SG est d'intégrer l'ensemble des apprentissages (linguistiques, discursifs et culturels) dans leurs divers niveaux de complexité (connaissances déclaratives, procédurales et stratégiques), le tout finalisé par un projet commun motivant. Dès lors, une mise en situation jouée permet d'envisager la langue comme un outil et non plus comme un objet de savoir. Elle donne à l'apprenant la possibilité de manipuler le matériau linguistique et de se l'approprier en expérimentant ses hypothèses par l'observation des réactions linguistiques et socioculturelles de ses interlocuteurs soumis aux actions entreprises. Enfin, l'enseignant encadrant l'activité veille au déroulement du scénario tout en proposant des situations-problème en rapport avec le monde de référence. Il s'agit donc d'une activité collective favorisant les échanges inter-humains en L2 (2) dans un lieu-thème plus ou moins réaliste permettant un "bain" linguistique et socioculturel, une sorte 
d'immersion artificielle (3). Le "potentiel d'immersion" s'avère très important puisqu'il influence directement la qualité de la mise en situation, intimement liée à l'évolution de l'identité fictive, à l'impact des situations-problème, ainsi qu'à l'adhésion de l'apprenant au lieu-thème. La SG met également l'accent sur l'importance de la créativité de l'apprenant. Elle est d'abord langagière, car l'apprenant se trouve dans une situation de production en L2 mais elle s'exprime également par la construction progressive de son personnage, et par la co-construction et l'évolution du lieu-thème.

\subsection{Transposer la Simulation Globale?}

Concevoir un dispositif informatique censé transposer la pratique de la SG telle que nous venons de la décrire semble une gageure tant il est probable que nous serons amenés à adapter ses fondements, voire à les dénaturer. La réification de l'approche communicative en un environnement informatique nécessite la création d'une tâche linguistique qui part de données riches et authentiques (Mangenot, 1998), qui prévoit des interactions variées, et qui fait référence à la "socioculture" cible. Il s'agit de rendre l'apprenant actif, responsable de son apprentissage en lui proposant des situations où il peut mettre à l'essai ses hypothèses. De ce principe, proche de la "pédagogie active" d'Adolphe Ferrière et basé sur la "théorie de l'intelligence" de Jean Piaget, sont nés les micromondes (Papert, 1980). Leurs évolutions et le recours à la réalité virtuelle ont permis d'augmenter le sentiment de réalisme susceptible de mobiliser et de contextualiser la parole, ce qui s'avère particulièrement intéressant dans une optique d'enseignement des langues étrangères. La représentation symbolique de l'apprenant interagissant dans le monde virtuel (avatar) facilite la construction de son identité fictive. Si l'environnement supporte des connexions simultanées, le cadre peut alors être partagé en temps réel par l'ensemble des personnes connectées et accueillir une activité collective organisée de manière coopérative ou collaborative. Dans la formation en langue, la collaboration est souvent privilégiée car, définie comme une activité coordonnée et synchronisée résultant d'une tentative continue pour construire une conception partagée du problème (Dillenbourg et Baker, 1996), elle suscite davantage d'interactions (Henri et Lundgren-Cayrol, 2001). En ce qui concerne la créativité, il sera intéressant de pouvoir intégrer et sauvegarder des productions (écrites, graphiques, etc.) des apprenants afin de leur permettre de contribuer à l'évolution de l'environnement (co-construction du lieu-thème). Quant à la créativité langagière de l'apprenant (production en L2), elle sera encouragée par le rassemblement des participants disposant des outils de communication et des aides adéquats, ainsi que par la cohérence des situations-problème. Pour aider la vérification et l'ajustement des hypothèses émises par les apprenants, l'observation des actions effectuées par les intervenants, y compris leurs réactions langagières, devra s'effectuer en temps réel. Ainsi, les interactions synchrones devront être majoritaires et envisagées dans le cadre de scénarios collaboratifs. Plus l'environnement fournira de la rétroaction immédiate, plus la vérification des hypothèses sera aisée, et plus la créativité langagière des apprenants sera encouragée. Pour un meilleur contrôle et ajustement des événements, pour une rétroaction davantage personnalisée, ainsi que pour la qualité des échanges porteurs d'apprentissage (négociations, co-construction des connaissances, etc.) (Deaudelin et Dubé, 2003), l'intervention humaine semble décisive. Par conséquent, les environnements de simulation en réseau devront prévoir un encadrement humain, soutenu éventuellement par les agents logiciels intervenant dans des tâches simples et récurrentes.

Communication et collaboration entre les participants, niveau/degré de perception (réalisme), sollicitation de la créativité des apprenants (identité, lieu-thème, langage) et accompagnement (tutorat) sont les critères au travers desquels nous allons examiner quatre environnements.

\section{Quatre environnements}

Les environnements évoqués dans ce paragraphe se donnent pour objectif de développer des compétences langagières et communicatives, en s'inspirant de la SG. Ces environnements se distinguent par leurs ergonomies, leurs scénarios pédagogiques et leurs choix technologiques. Il s'agit de SimuLigne, du MOO 
Français, d'Exills et de TLTS. Précisons que tous ces dispositifs, excepté TLTS, sont dédiés à la pratique ou à l'apprentissage du FLE.

SimuLigne (Chanier, 2002), (Reffay et al., 2002) est une activité collaborative intégrée dans la plateforme de téléformation WebCT. La situation-problème de départ consiste en l'élaboration collaborative d'une ville virtuelle par des groupes d'une dizaine d'apprenants, chaque groupe étant encadré par un tuteur humain. Ensuite, les membres du groupe jouent les personnages en les faisant intervenir dans des situations conventionnelles ou inattendues. La plate-forme intègre un ensemble de logiciels qui permettent de mettre en ligne des documents, de communiquer de façon synchrone et asynchrone, de gérer un agenda collectif, de créer des espaces de travail individuels et collectifs, d'utiliser des procédures spécifiques pour soumettre des travaux, les corriger ou les partager avec d'autres. Si les apprenants ne sont pas représentés par des avatars, les concepteurs ont eu le souci de faire en sorte que chaque participant (apprenant ou tuteur) puisse se créer un sentiment d'espace privé dans son espace individuel, mais également gérer les aspects émotionnels à travers les contraintes de la plate-forme, et se créer une image mentale des autres participants (Chanier, 2002).

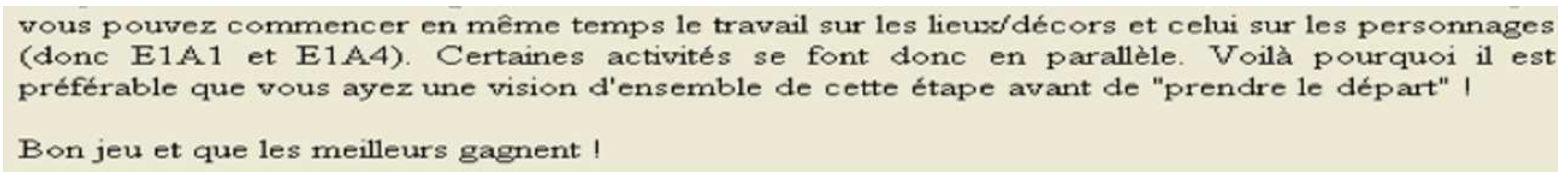

\section{Renseignements pratiques}

$\begin{array}{lr}\text { Date de mise en ligne } & 11 \text { mai } \\ \text { Date de fin } & 1 \text { er juin }\end{array}$

\section{Activités}

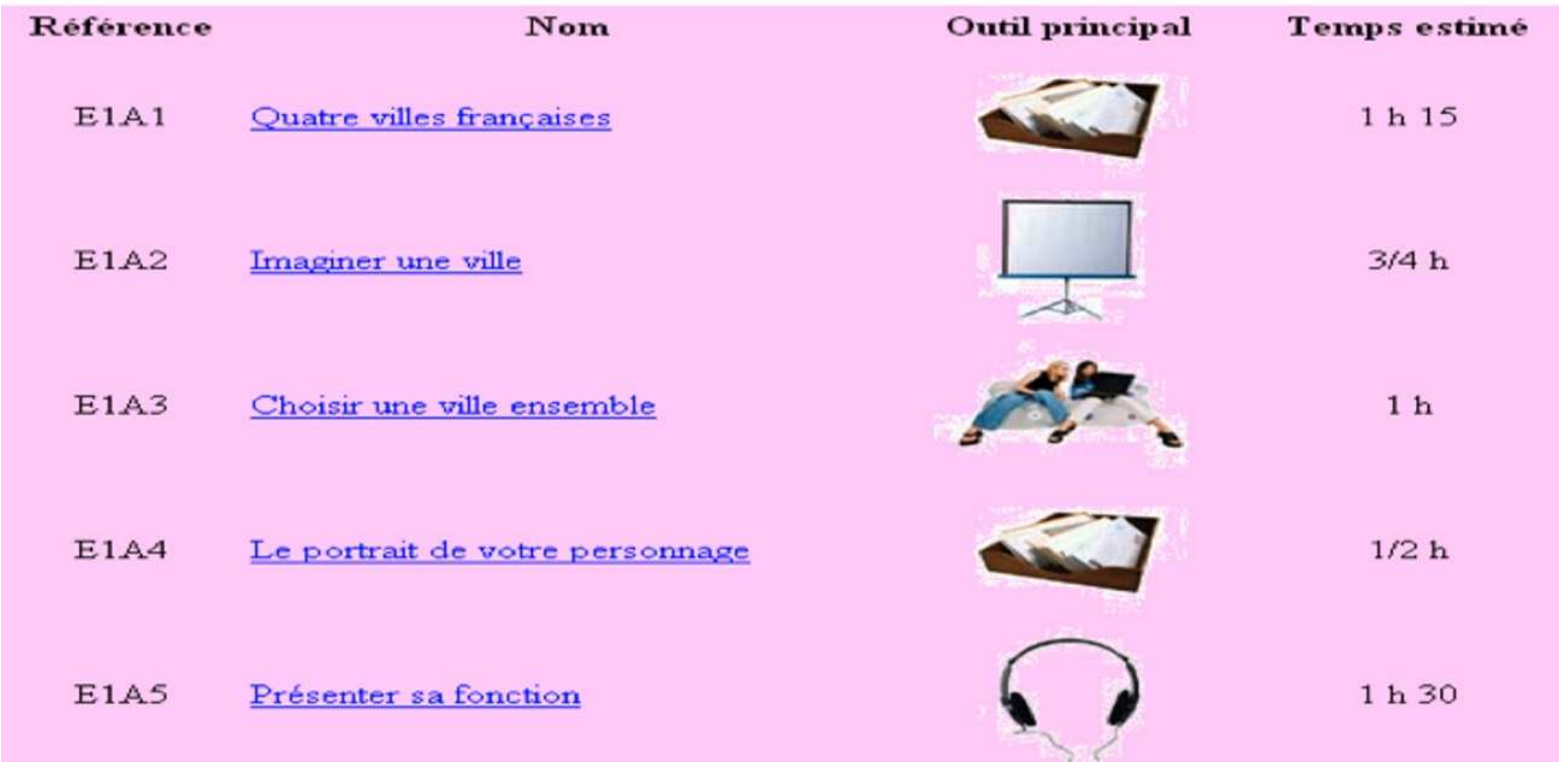

Figure $1 \cdot$ Ecran d'accueil de SimuLigne

Le MOO Français (MOOFrançais, 1999) est avant tout un MOO ${ }^{(4)}$, c'est-à-dire une application en réseau à laquelle on accède par Telnet, et qui permet de communiquer de façon textuelle avec d'autres participants dans le cadre d'un espace virtuel partagé. Les personnes connectées endossent une identité fictive, peuvent suivre un itinéraire, visiter des lieux, regarder des objets, converser en temps réel par écrit et contribuer à la construction de cet univers virtuel. C'est en quelque sorte de la "réalité virtuelle textuelle". Le MOO Français a pour cadre une représentation virtuelle de Paris, avec ses rues, ses musées, ses magasins. Nous pouvons y découvrir toute sorte de personnages, d'objets et de documents. Ce type 
d'environnements permet de mettre en place des activités qui s'apparentent à de la SG. Toutefois l'activité n'est pas a priori structurée pédagogiquement, ni encadrée. Aucune situation-problème ne préexiste aux connexions des utilisateurs, ces dernières étant créées et gérées par les intervenants et selon l'opportunité du moment. Ceci dit, les MOO sont des environnements utilisables (et utilisés) pour pratiquer et apprendre une langue étrangère (Turbee, 1996), (Schwienhorst, 1997).

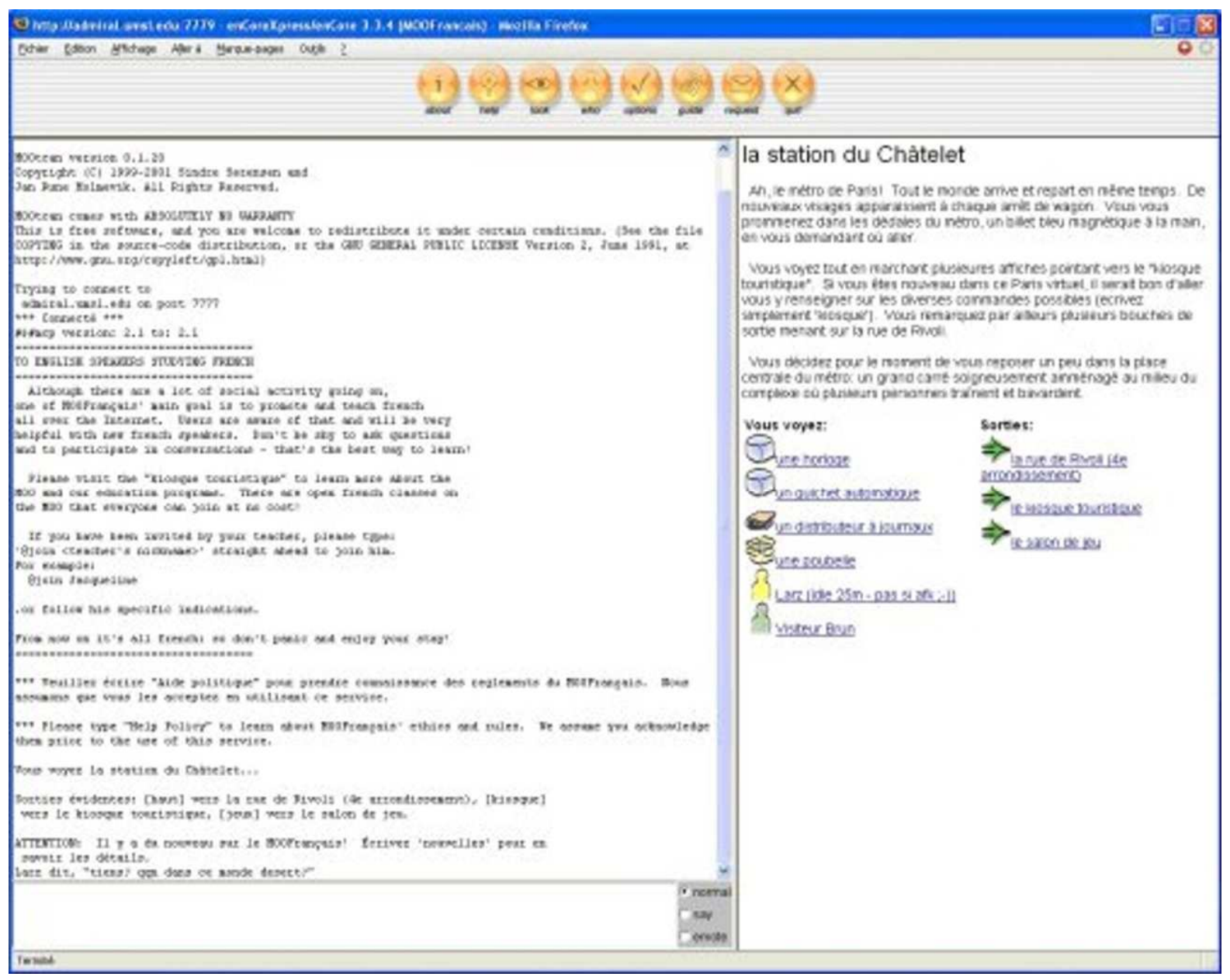

Figure $2 \cdot$ Interface web du MOO Français

La plate-forme Exills (Segond et al., 2005), (Brun et al., 2002), (ExillsHomePage, 2002) quant à elle, associe les principes du MOO et du travail collaboratif, tout en proposant une visualisation en 3D qui met en scène l'apprenant pour l'amener vers les tâches linguistiques. Les apprenants naviguent dans un décor virtuel dans lequel ils doivent accomplir des tâches professionnelles tout en interagissant entre eux, avec des tuteurs humains qui peuvent intervenir ponctuellement, notamment lors des activités de production libre, ou encore avec des agents conversationnels animés. La navigation dans le monde est apparemment libre mais l'enchaînement des activités a été contraint. Par exemple, les apprenants doivent préparer un exposé sur une des technologies issues de XRCE ${ }^{(5)}$ et le présenter aux autres apprenants lors d'une réunion. Puis, ils partent à la recherche d'un client à qui ils doivent vendre la technologie présentée lors de leur exposé. Même si l'objectif avoué est de scénariser les technologies linguistiques et collaboratives de XRCE de façon attractive afin de les présenter aux professionnels (Parmentier, 2002), la plate-forme Exills est un véritable environnement dédié à l'apprentissage des langues. 


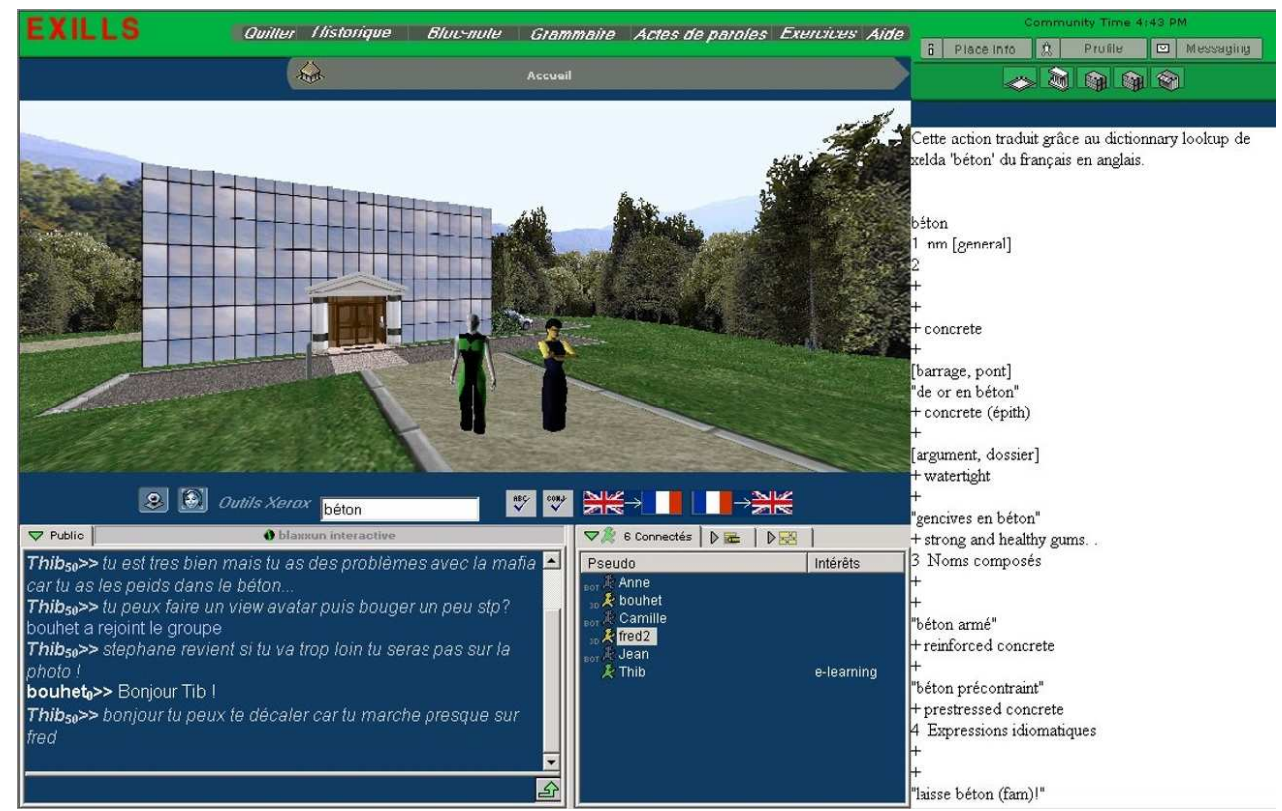

Figure 3 • Interface web d'Exills

Enfin, TLTS (Johnson et al., 2004), (Johnson et al., 2005), (TLTSHomePage, 2004) est un jeu vidéo 3D conçu pour l'armée des Etats-Unis dont l'objectif est de faire acquérir aux militaires un minimum de compétences communicatives et culturelles avant d'aller en mission. Le "joueur" progresse dans une mission de reconnaissance tout en communiquant oralement avec des agents virtuels autonomes représentant des autochtones. Un module tuteur analyse en temps réel les actions de l'apprenant afin de l'aider au niveau de sa mission, de ses productions orales, et de son comportement. La qualité de sa réalité virtuelle, son intelligence artificielle, l'intégration des modalités orale et gestuelle, le réalisme des situations présentées, ainsi que son environnement-auteur font de TLTS un bon outil d'apprentissage de la langue orienté par la tâche.

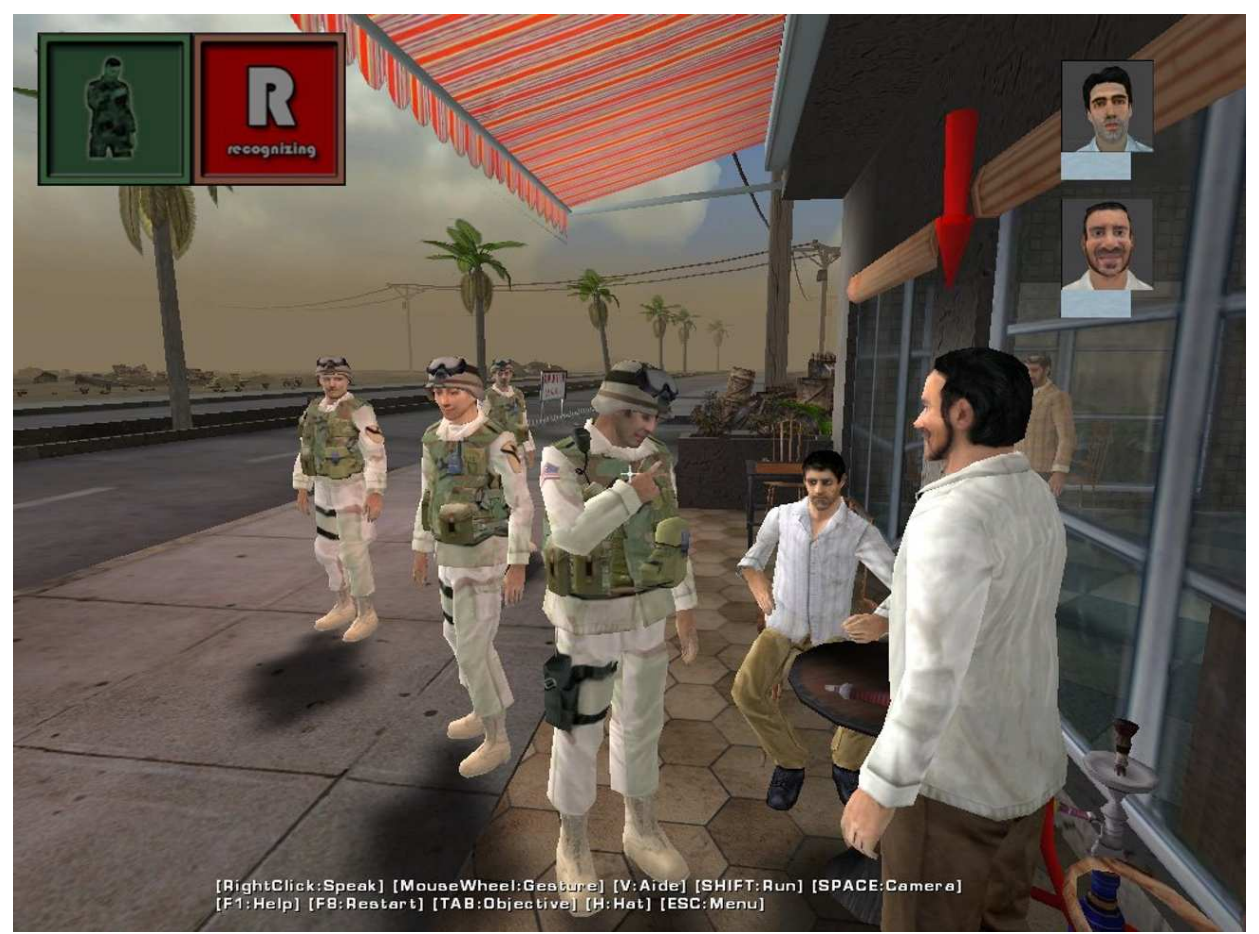

Figure 4 • Copie d'écran de TLTS 
Ces projets ont tous pour ambition de développer des compétences communicatives en se fondant sur des simulations mais ils reposent sur des scénarios très divers, impliquent des technologies fort différentes, sont plus ou moins réalistes, et leurs coûts de développement ne sont pas les mêmes. Partant de cet état de l'art, nous proposons une amorce de typologie des environnements informatiques dédiés à la pratique communicative d'une langue étrangère. Les deux premiers critères concernent des différences techniques, les critères suivants regroupent les éléments de la simulation globale retenus pour sa transposition en réseau.

\begin{tabular}{|l|l|l|l|l|}
\hline & SimuLigne & MOO Fr & Exills & TLTS \\
\hline \hline Type de logiciel & site web & MOO & client léger & jeu vidéo \\
\hline \hline Technologie & html & telnet / web & html + vrml & 3D + vocale \\
\hline \hline Communication & réelle & libre & réelle & simulée \\
\hline \hline Collaboration & guidée & libre & guidée & aucune \\
\hline \hline Perception & limitée & limitée & forte & immersive \\
\hline \hline Créativité & monde + lang. & monde + lang. & langagière & langagière \\
\hline \hline Tutorat & humain & non supporté & mixte & machine \\
\hline \hline
\end{tabular}

Tableau 1 - Une typologie des environnements

Tous ces dispositifs visent la communication, mais de façons fort différentes. Si les échanges sont stéréotypés et réduits on ne peut parler que d'une pseudo-approche communicative. François Mangenot parle même d'une prétendue approche communicative consistant pour l'apprenant à jouer (simuler) des situations calquées sur la vie réelle (Mangenot, 1996). Il est notamment question de la répétition/reproduction fidèle (dramatisation d'un dialogue préexistant, canevas dialogique fortement stéréotypé) qui limite la créativité de l'interlocuteur et diminue l'authenticité des enjeux communicatifs (insuffisance d'ancrage et d'épaisseur des identités fictives, absence de production libre et d'improvisation). Si l'environnement n'envisage que des communications avec agents logiciels, celles-ci seront nécessairement balisées et donc réductrices. C'est ce qui explique le "simulée" du critère "communication" de l'environnement TLTS. Inversement, si la communication entre les interlocuteurs humains s'effectue uniquement de manière libre (comme dans un MOO) sans aucune contrainte de thème ou de forme, il est fort possible qu'elle n'améliore pas leur performance de façon significative. L'efficacité des échanges se détermine par leur adéquation au contexte, au type et à la finalité de discussion dans laquelle ils apparaissent (Deaudelin et Dubé, 2003). Dans la plupart des plates-formes, c'est la tâche à accomplir par les apprenants qui délimite, dans un premier temps, les thèmes de leurs interactions.

La richesse des interactions, ainsi que la forme du travail envisagée dans l'environnement informatique, restent en étroit rapport avec la dimension collective de l'activité. Les activités et parcours mis en place dans l'environnement TLTS sont individuels, ce qui exclut les interactions inter-humaines. En revanche, Exills et SimuLigne alternent des formes collaboratives (partage d'une même tâche par l'ensemble des participants) et coopératives (division des sous-tâches). De manière générale, chaque dispositif supportant des connexions multiples permet toute sorte de composition (hétéro/homogène). Parmi les dispositifs retenus, SimuLigne et le MOO recourent à des connexions croisées entre les natifs et les non-natifs (éventuellement de L1 différentes) recréant ainsi une classe virtuelle hétérogène. 
La perception du cadre, de soi-même, des autres participants, de leur activité est également une question importante (quoiqu'artificielle puisqu'apportée par l'usage du dispositif). La majorité des environnements cités tentent d'offrir un cadre plus ou moins réaliste, cherchant ainsi à plonger l'apprenant dans une "réalité simulée" de la société cible. En effet, la quantité et la qualité des représentations, informations et indices perceptibles par les apprenants peuvent conditionner leur implication et leur motivation. En se basant sur l'importance des représentations que l'apprenant a de sa propre image en tant qu'apprenant (effet Pygmalion), des auteurs comme (Arnaud et Serdidi, 2003) émettent l'hypothèse que dans un environnement virtuel, l'apprenant représenté par un avatar peut repousser ses propres limites et améliorer ses résultats en effectuant inconsciemment un transfert des caractéristiques de l'avatar à sa propre personne et générer un processus d'apprentissage. Si le MOO Français ne propose aucune représentation symbolique de l'apprenant, SimuLigne prévoit la construction des portraits des participants alors qu'Exills et TLTS recourent à une représentation humanoïde en 3D.

La question du tutorat est abordée par ces dispositifs de manière très différente selon les possibilités offertes par le type de logiciel et le scénario pédagogique. Toutefois, en alternant le tutorat humain et le tutorat système, la plate-forme Exills semble trouver une complémentarité entre la technologie et l'intelligence humaine.

Comme le démontrent les environnements cités, la transposition des différentes caractéristiques de la SG s'avère réalisable seulement dans une certaine mesure, souvent limitée par les technologies employées. Il est alors question de consentir à un compromis en se concentrant davantage sur certains éléments que sur d'autres. En utilisant une plate-forme de téléformation existante, SimuLigne se focalise sur l'apprentissage collaboratif et propose des outils et un scénario pédagogique allant dans ce sens. L'environnement, s'il reste peu immersif, permet l'intégration aisée des productions des apprenants. A l'inverse, en intégrant des capacités de dialogue oral dans une réalité virtuelle en 3D, TLTS se focalise sur le degré de perception et sur le réalisme des situations communicatives. Toutefois, le dispositif n'envisage pas d'activités d'apprentissage collaboratif et n'induit que la seule créativité langagière des apprenants isolés.

Etant donné la situation d'apprentissage et le public cible concerné dans cet article, aucun des dispositifs ne semble réunir tous les éléments identifiés comme cruciaux pour une ouverture virtuelle du milieu hétéroglotte. Exills, le plus complet sur ce point, s'avère néanmoins peu accessible à des modifications de ses contenus et le coût de sa conception reste élevé.

\section{La plate-forme MEPA-2D}

En concevant MEPA-2D, nous cherchions des moyens permettant de compenser une faible expérience socioculturelle et de neutraliser un certain conventionnalisme de l'usage de la langue cible dans le milieu hétéroglotte. Notre objectif était de concevoir un nouvel environnement inspiré de la simulation globale pour la pratique et l'apprentissage du FLE, sans avoir à mettre en œuvre des technologies complexes ou onéreuses. Les environnements qui associent les principes du MOO/des mondes virtuels et ceux de la réalité virtuelle se multiplient (Jones, 2004), (Jones et al., 2004). Ceci dit, ces environnements très immersifs imposent un savoir-faire spécifique (programmation 3D) qui interdit la conception de nouveaux mondes et scénarios aux non-spécialistes. De plus, ils imposent des machines relativement puissantes (processeur, mémoire, carte graphique) si on veut atteindre un niveau de réalisme satisfaisant. Thibault Parmentier (du projet Exills) précise que la réalité virtuelle est réputée coûteuse en terme de bande passante, gourmande en puissance calcul, complexe pour ce qui est de la prise en main de l'utilisateur ou tout simplement chère à la réalisation (Parmentier, 2002). La plus-value didactique apportée par ces technologies doit être appréciée au regard de leurs coûts élevés. Or, nous estimons que le rapport plus-value/coût est encore trop faible. C'est la raison pour laquelle nous avons cherché à mettre en œuvre une technologie qui permette de concevoir facilement et à faible coût des lieux virtuels et des 
activités qui y prennent place.

\subsection{Les mondes virtuels dans MEPA-2D}

MEPA-2D (MEPAHomePage, 2006) est un environnement logiciel client-serveur qui permet à des apprenants, à des enseignants, et à des agents logiciels ${ }^{(6)}$, d'agir et d'interagir à distance dans un monde virtuel visualisé en 2D manipulable à la souris. Un monde virtuel est un ensemble de scènes (lieux, vues, etc.) reliées entre elles par des passages (directions, issues, portes, etc.). Ce monde peut être modélisé à l'aide d'un graphe orienté connexe dans lequel les sommets représentent les scènes, et les arcs les passages, avec ou sans précondition. Les conditions de passage d'une scène à une autre permettent d'implémenter des stratégies d'évaluation non-intrusives, c'est-à-dire sans interruption du déroulement du scénario. Un monde virtuel MEPA-2D possède la même structure que le monde qu' on peut explorer dans un MOO, mais il est rendu à l'écran à l'aide de fonds photographiques, d'objets et d'avatars en 2D. Des ambiances et des effets sonores viennent s'ajouter afin d'augmenter le sentiment de réalisme.

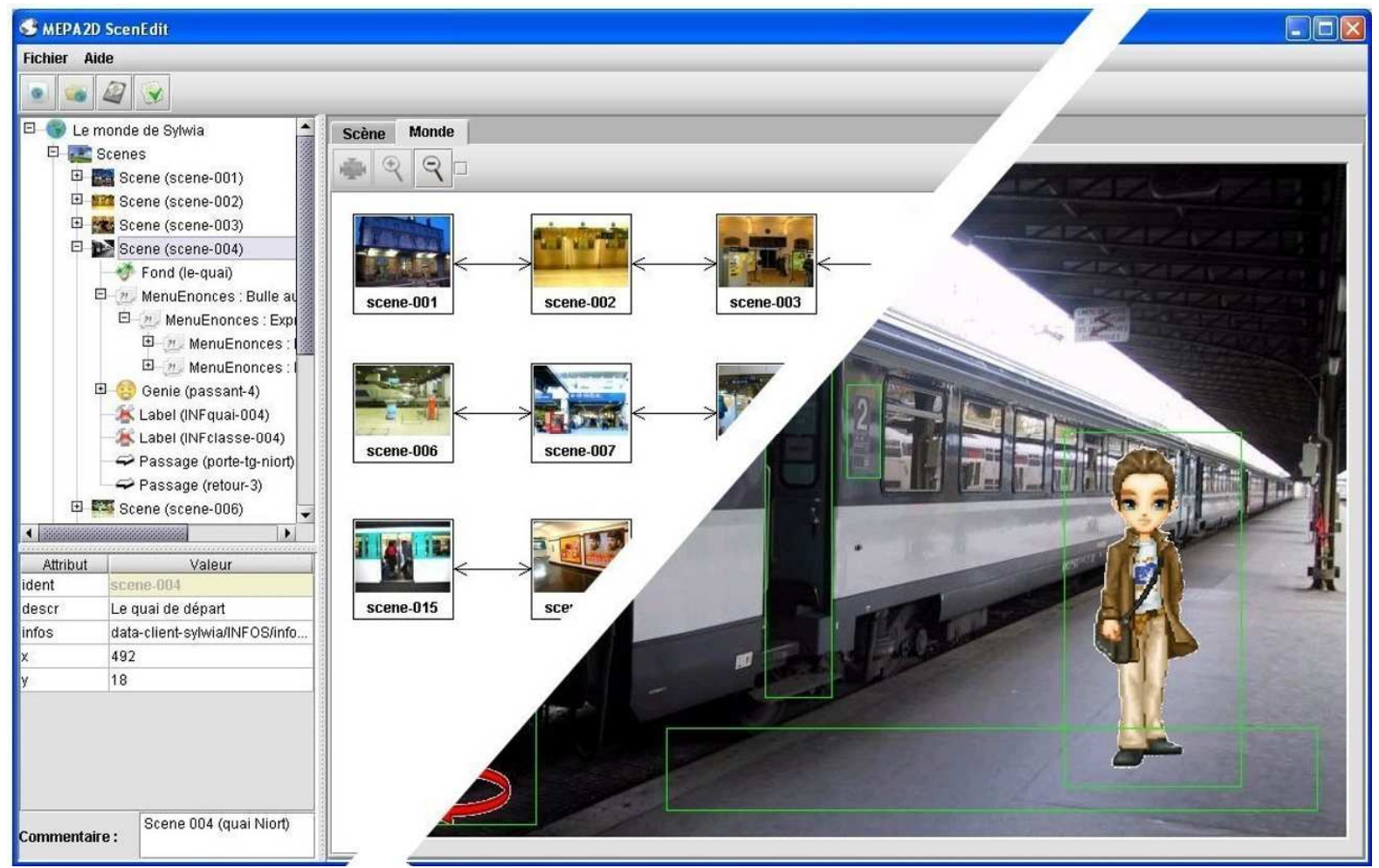

Figure 5 • Vues de l'éditeur de scènes de MEPA-2D

Pour réaliser un nouveau monde MEPA-2D, un appareil photo et un dictaphone numériques suffisent. Les scènes sont ensuite décrites sous la forme d'un fichier XML et validées par une DTD. Pour faciliter la création des mondes virtuels, nous avons développé un petit éditeur graphique (cf. figure 5) qui permet de composer et d'éditer des scènes à la souris sans rentrer dans le code XML. Les enseignants peuvent ainsi construire un monde virtuel, en accord avec un scénario, sans connaissances particulières en informatique.

La description du monde virtuel, totalement déclarative, est stockée au niveau du serveur sous la forme de fichiers XML. Les différentes ressources sont stockées au niveau des clients afin de minimiser les temps de chargement et de communication entre le serveur et les clients, ce qui autorise les connexions à faible débit. Dès qu'un client se connecte au serveur, il visualise un monde en deux dimensions dans lequel il est représenté par un avatar. Les personnes connectées peuvent se rencontrer et interagir au travers de leurs avatars. Plus précisément, ils peuvent : 
- Déplacer leur avatar (déplacements intra-scène et inter-scènes) ;

- Prendre et déposer des objets (notion d'inventaire / de panier);

- S'échanger des objets afin de simuler des transactions ;

- Changer d'apparence afin d'exprimer des changements d'humeur ;

- Communiquer par écrit dans les scènes au travers de "bulles" type BD ;

- Communiquer par écrit au travers d'une messagerie instantanée ;

- Consulter des ressources externes (pages web) à partir des objets du monde, etc.

Les apprenants peuvent également consulter les objets placés dans les scènes pour connaître leur nom en français ou accéder à une ressource externe attribuée à l'objet. Il s'agit en général de documents authentiques iconiques, textuels, ou audiovisuels, particulièrement appréciés dans les milieux hétéroglottes où sa relative pénurie limite le contact avec la "socioculture" cible. Quant aux agents logiciels, ils ne sont pour l'instant que de simples agents conversationnels qui réagissent aux énoncés des apprenants seulement si ces derniers restent en rapport avec le canevas et la situation de communication prévue dans la scène. Certaines interactions déclenchent des comportements simples comme l'échange d'objets (cartes bancaires, titres de transport, etc.) ce qui permet à l'apprenant de vérifier la force illocutoire de ses énoncés. La présence des agents, spécifique à des scènes données, a pour but de baliser le parcours de l'apprenant en lui fournissant des informations nécessaires pour la poursuite de son activité. Ils peuvent également fournir une rétroaction minimum, appréciée notamment en cas d'indisponibilité du tuteur humain.

Les traces de l'activité des différents acteurs (apprenants, tuteurs, agents logiciels) sont stockées au niveau du serveur sous la forme de fichiers XML facilement exploitables. Chaque événement contient l'instant (date et heure) et le lieu où il a été produit, l'identificateur de l'acteur, son rôle, le type de l'action, et une description détaillée. Pour l'instant, nous utilisons des feuilles de style XSLT pour transformer les fichiers de traces (visualisations, extractions, tris, statistiques, etc.). Des modules de traitements automatiques des traces viendront compléter la boite à outils à disposition des tuteurs et pédagogues.

\subsection{Interface et fonctionnalités du client MEPA}

L'interface graphique d'un client venant de se connecter au serveur se construit en fonction des droits associés au profil. Pour l'instant, nous n'avons défini que deux profils : apprenant et tuteur. L'interfaceapprenant contient trois onglets :

- L'onglet [ monde virtuel ] contient la scène en cours et permet de réaliser les actions décrites précédemment.

- L'onglet [ calepin ] permet la prise de notes, sauvegardées et rechargées à chaque connexion. Cet outil autorise le copier-coller vers le champ de saisie des énoncés libres des apprenants.

- L'onglet [ messagerie ] contient une messagerie instantanée qui permet de contacter un participant (un seul à la fois), quelle que soit la scène où il se trouve.

L'environnement prévoit également l'accès permanent à un dictionnaire en ligne, un conjugueur ainsi qu'un dictionnaire thématique reprenant et développant les actes de paroles disponibles depuis les scènes du monde. L'interface-tuteur contient les trois onglets de l'interface-apprenant plus un explorateur :

- L'onglet [ explorateur ] a plusieurs fonctions : il fournit des informations sur les scènes et une aide à l'assistance (actions des participants, possibilités d'interventions des tuteurs, 
mots-clés identifiables par les agents artificiels, etc.), il permet aussi de se "téléporter" dans n'importe quelle scène du monde virtuel sans avoir à utiliser les passages.

De plus, les tuteurs ont des possibilités supplémentaires :

- Ils peuvent "voir sans être vus". Ils peuvent ainsi observer l'activité des apprenants, puis apparaître sous une apparence adéquate (personnage du scénario, passant, etc.).

- Ils peuvent se "téléporter" vers la scène d'un autre participant puis intervenir de leur propre chef sous une des apparences à leur disposition.

- Ils peuvent intervenir à la place d'un agent logiciel. Cela permet de varier le comportement des agents, voire pallier aux éventuelles insuffisances de leur moteur conversationnel.

Dans une scène, les interactions dialogiques reposent sur l'utilisation de "bulles" comme dans une bande dessinée. Deux méthodes sont possibles pour créer une bulle : soit on utilise directement le champ de saisie "nouvelle bulle" et on tape un énoncé, soit on passe par un menu "bulle automatique" si la scène le permet. En effet, chaque scène peut offrir un ensemble structuré d'énoncés prévus à l'avance en fonction du scénario ou des règles de savoir-vivre. De plus, ces énoncés peuvent être modifiés par les apprenants avant d'être validés.
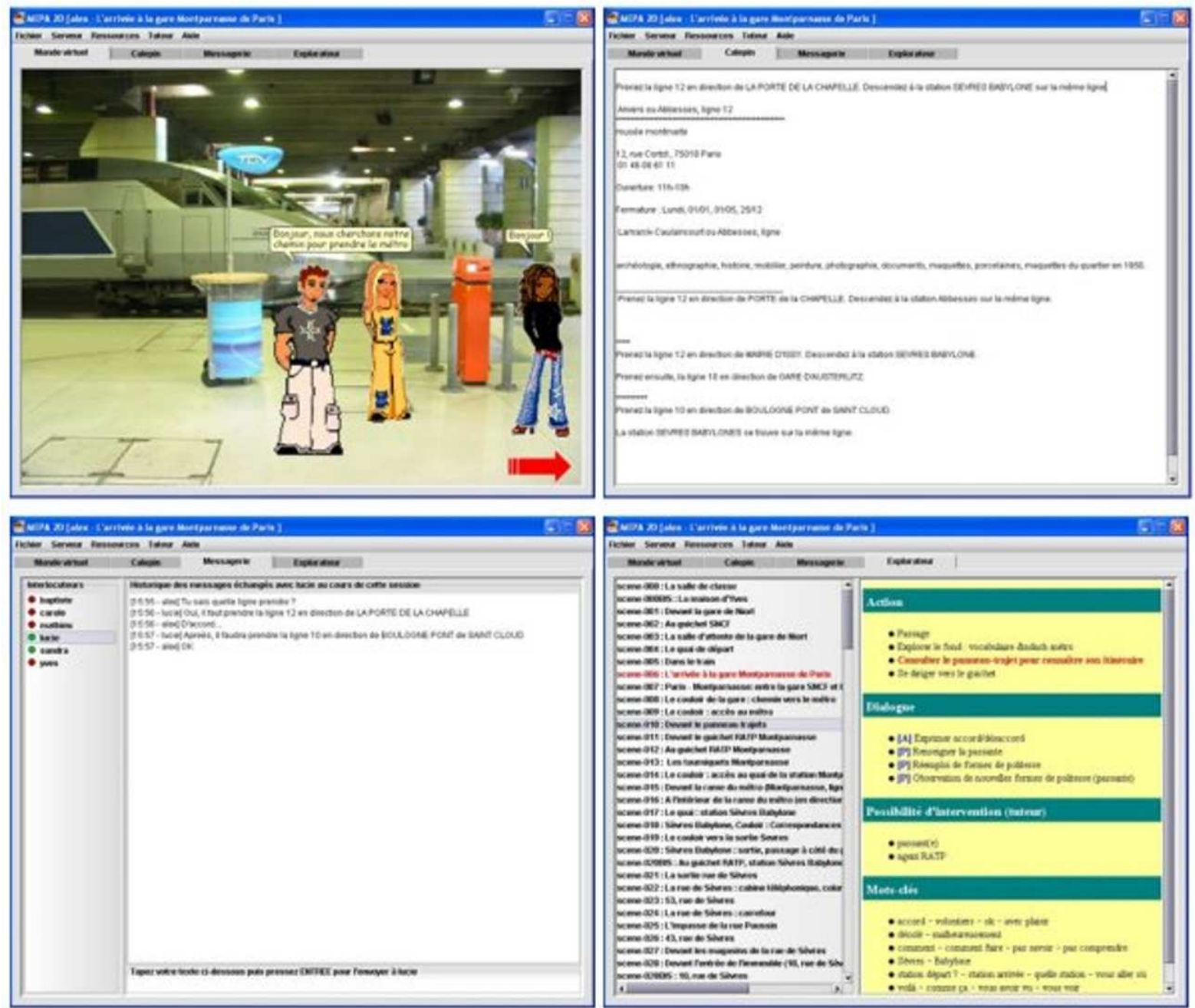

Figure $6 \cdot$ Onglets du client MEPA (interface-tuteur) 


\begin{tabular}{|l|l|l|l|l|l|l|}
\hline \multicolumn{2}{|l|}{ MEPA-2D } \\
\hline \hline Logiciel & Techno. & Comm. & Collab. & Perception & Créativité & Tutorat \\
\hline \hline $\begin{array}{l}\text { jeu } \\
\text { vidéo }\end{array}$ & Java 2D & réelle & guidée & forte & langagière & mixte \\
\hline \hline
\end{tabular}

Tableau 2 • La plate-forme MEPA-2D selon notre typologie

\section{Scénarisation pour MEPA-2D}

L'environnement MEPA-2D est une plate-forme logicielle vide de contenu. Pour l'utiliser, il faut concevoir un scénario en fonction d'un public cible, d'une situation d'apprentissage concrète et d'objectifs didactiques. Enfin, il faut développer le monde virtuel (structure et ressources) qui supporte le scénario. Les sous-sections suivantes présentent des contextes d'usage du dispositif ainsi qu'un premier scénario.

\subsection{Contextes d'usage du dispositif}

Dans l'absolu, le dispositif permet de mettre en contact un certain nombre de participants, voire des classes entières. Ainsi, son encadrement peut être assuré par les enseignants des classes respectives sans la nécessité de mobiliser de ressources humaines supplémentaires. Plusieurs contextes d'usage sont alors envisageables. L'activité collective peut impliquer par exemple des classes concernées par un programme plus large de coopération scolaire (projet de jumelage, échanges et séjours linguistiques, etc.), issues des différents milieux hétéroglottes. Selon les disponibilités des classes concernées, l'activité peut prendre la forme de séances thématiques synchrones en rapport avec le programme suivi en présentiel, ou avec le sujet de coopération scolaire. Si les contraintes de disponibilités simultanées des classes sont trop importantes, la simulation peut être réalisée dans le cadre d'entraînement pratique en langue, cette fois-ci en dehors des cours en présentiel (sorte de cours particuliers). L'activité peut également s'ouvrir à d'autres disciplines et prendre forme d'une propédeutique, ce qui a été déjà encouragé par Francis Yaiche (Yaiche, 1996). Afin d'impliquer plus facilement des natifs, les simulations peuvent avoir lieu entre une classe de langue sur objectifs spécifiques (FOS) issue du milieu hétéroglotte et une classe de natifs en formation professionnelle correspondant au domaine socioprofessionnel de la simulation. Par ailleurs, différentes classes de FLE engagées dans une simulation globale en présentiel peuvent recourir à l'usage ponctuel de MEPA-2D afin d'organiser des séances synchrones. Cette situation d'apprentissage s'approche alors de celle des simulations en ligne asynchrones (Hôtel, 1996), (Village, 2006) proposant tout de même une perception et contextualisation plus importantes.

\subsection{Première scénarisation}

Pour notre première scénarisation pédagogique de simulation "généraliste", nous nous sommes concentrés sur les marqueurs des relations sociales (salutations, formes d'adresse, convention de prise de parole), la familiarisation avec les règles de politesse (remerciements, expressions de regret, etc.) et la sensibilisation aux différents registres de langue dans des situations simples de la vie quotidienne (parole fonctionnelle). En prenant en compte les principaux besoins du public cible (non-natifs du milieu hétéroglotte), nous avons cherché à enrichir son expérience socioculturelle et sa compétence sociolinguistique. Cette dernière, étant définie comme la capacité de reconnaître et de produire un discours socialement approprié en contexte (Conseil de l'Europe, 2001), s'avère particulièrement difficile à acquérir notamment en dehors de la société cible. Pour la faciliter, certains suggèrent d'avantager les cours de langue fonctionnelle (Lyster et Rebuffot, 2002) ou de compléter l'enseignement traditionnel par des interactions avec des natifs (Mougeon et al., 2002), car seule l'interaction authentique permet l'appropriation efficace 
de la variante sociolinguistique. Le cours de langue de type "télécollaboratif", permettant davantage d'interactions avec des natifs, accélérait le développement de cette habileté (Belz et Kinginger, 2002). Ces conditions sont remplies par la plate-forme MEPA-2D. Par ailleurs, l'emploi approprié des variantes sociolinguistiques dépend de plusieurs facteurs (âge et sexe des interlocuteurs, formalité de l'interaction, distance sociale et degré de familiarité des interlocuteurs) (Dewaele, 2002) dont la considération peut également être facilitée grâce aux possibilités offertes par MEPA-2D. La représentation iconique des personnages aidera la perception des éléments liés à l'interlocuteur (âge, sexe), la visualisation du cadre socioculturel permettra de préciser le contexte et d'estimer la formalité de l'échange, alors que le degré de familiarité entre les interlocuteurs sera déterminé par le canevas de l'histoire virtuellement vécue par l'ensemble des apprenants et par les relations qui s'établiront entre eux et les personnages rencontrés tout au long du parcours.

Concrètement, nous avons développé un monde virtuel composé de plus de 150 scènes, chacune possédant sa propre ambiance sonore. Une enquête sur un personnage fictif constitue la trame du scénario impliquant une diversité des paysages socioculturels. Les apprenants sont amenés à observer des situations ritualisées et à participer à des échanges formels ou informels (commande dans un café ou restaurant, visite de courtoisie chez un particulier, démarches administratives, etc.). Ils doivent interpréter des indices et se concerter sur leur signification (négociations, discours argumentatif), effectuer des recherches documentaires afin de sélectionner des informations pertinentes, confronter leurs résultats avec ceux des autres apprenants, ou consulter un "personnage-référence" incarné par un tuteur. En termes de productions écrites, les apprenants sont bien entendu amenés à interagir de façon libre (à l'aide des "bulles" et de la messagerie), mais aussi à remplir des formulaires administratifs, à donner des interviews et à rendre compte de leur progression auprès du personnage-référence. Pour structurer le niveau pédagogique, nous définissons des types de scènes :

Dans les scènes de découverte les apprenants sont exposés à un nouveau matériau socioculturel et linguistique. Les interventions proactives des agents ont pour objectif d'attirer leur attention vers ces nouveaux éléments. Ils peuvent observer et participer aux échanges, s'informer sur la réalité socioculturelle, et manipuler les éléments linguistiques librement ou à partir d'actes de parole éditables. Depuis le menu de chaque avatar, les apprenants ont accès à un ensemble d'actes de parole dédiés à la situation de communication (menu "bulle automatique"). Le recours à ce type de communication facilite et structure l'échange, notamment avec les agents logiciels.

\begin{tabular}{|l|l|}
\hline $\begin{array}{l}\text { Eléments linguistiques : } \\
\text { Construction de } \\
\text { questions }\end{array}$ & $\begin{array}{l}\text { Tout au long du parcours, les apprenants sont confrontés } \\
\text { aux questions des personnages. } \\
\text { Depuis leur menu, les apprenants ont accès à des } \\
\text { questions de plus en plus élaborées. }\end{array}$ \\
\hline \hline $\begin{array}{l}\text { Eléments } \\
\text { sociolinguistiques : } \\
\text { Tutoiement et } \\
\text { vouvoiement }\end{array}$ & $\begin{array}{l}\text { Echange dans une scène de découverte (extrait) : } \\
\text { Alex (bulle automatique : Saluer) : Enchanté de vous } \\
\text { connaître ! } \\
\text { Yves : Ah non ! Ne me dis pas "vous" ! Nous nous } \\
\text { connaissons et nous avons le même âge ! Tu peux me } \\
\text { dire "tu", d'accord ? } \\
\text { Alex (bulle automatique : Accord) : Avec plaisir ! } \\
\text { Yves : Très bien ! Des questions ? }\end{array}$ \\
\hline \hline $\begin{array}{l}\text { Eléments } \\
\text { socioculturels : } \\
\text { Eléments d'une gare } \\
\text { SNCF }\end{array}$ & $\begin{array}{l}\text { Scène de la gare Montparnasse (cf. figure 7) : } \\
\text { Observation de l'organisation d'une gare avec la } \\
\text { possibilité d'interroger différents éléments afin de } \\
\text { connaître le lexique : bornes pour composter les billets, } \\
\text { trains, quai, etc. }\end{array}$ \\
\hline \hline
\end{tabular}




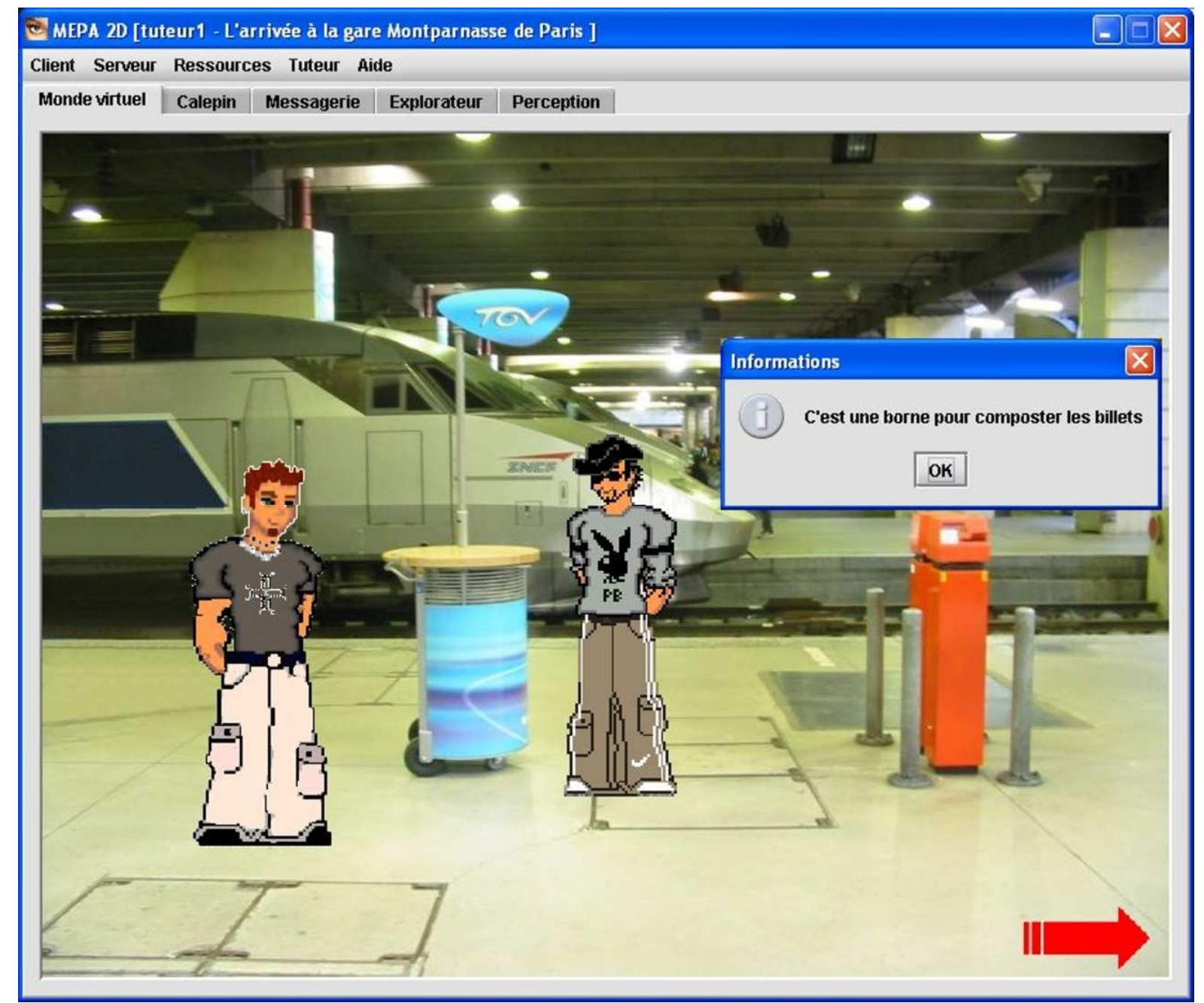

Figure 7 • Scène de la gare Montparnasse (scène de découverte)

Dans les scènes d'entraînement les apprenants sont généralement interpellés par les agents, les échanges étant dirigés vers des éléments présentés dans les scènes de découverte. Il est alors possible de vérifier la compréhension des réalités socioculturelles et de s'entraîner en interagissant. Les échanges prévus avec les agents logiciels sont construits de manière progressive (approfondissement du sujet, recours à un registre plus familier, formes grammaticales et lexicales plus complexes, etc.).

\begin{tabular}{|c|c|}
\hline $\begin{array}{l}\text { Eléments sociolinguistiques: } \\
\text { Tutoiement et vouvoiement } \\
\text { Niveaux de langage }\end{array}$ & $\begin{array}{l}\text { Scène du guichet SNCF (cf. figure } 8 \text { ) : } \\
\text { Alex : Salut monsieur } \\
\text { Guichetier : Pardon ??? Nous nous connaissons ??? } \\
\text { Bonjour ! Que puis-je pour vous? }\end{array}$ \\
\hline $\begin{array}{l}\text { Eléments socioculturels : } \\
\text { Déplacements dans le métro }\end{array}$ & $\begin{array}{l}\text { Echange dans une scène d'entraînement (extrait) : } \\
\text { Lucie (bulle automatique) : Pourriez-vous nous aider, } \\
\text { s'il vous plaît? } \\
\text { Agent de propreté : Bien sûr ! Qu'est-ce qui vous arrive } \\
\text { ? } \\
\text { Lucie (bulle libre) : Où est le métro ? } \\
\text { Agent de propreté : Vous cherchez le métro? Continuez } \\
\text { et suivez les panneaux "métro". Vous cherchez quelle } \\
\text { station? }\end{array}$ \\
\hline
\end{tabular}




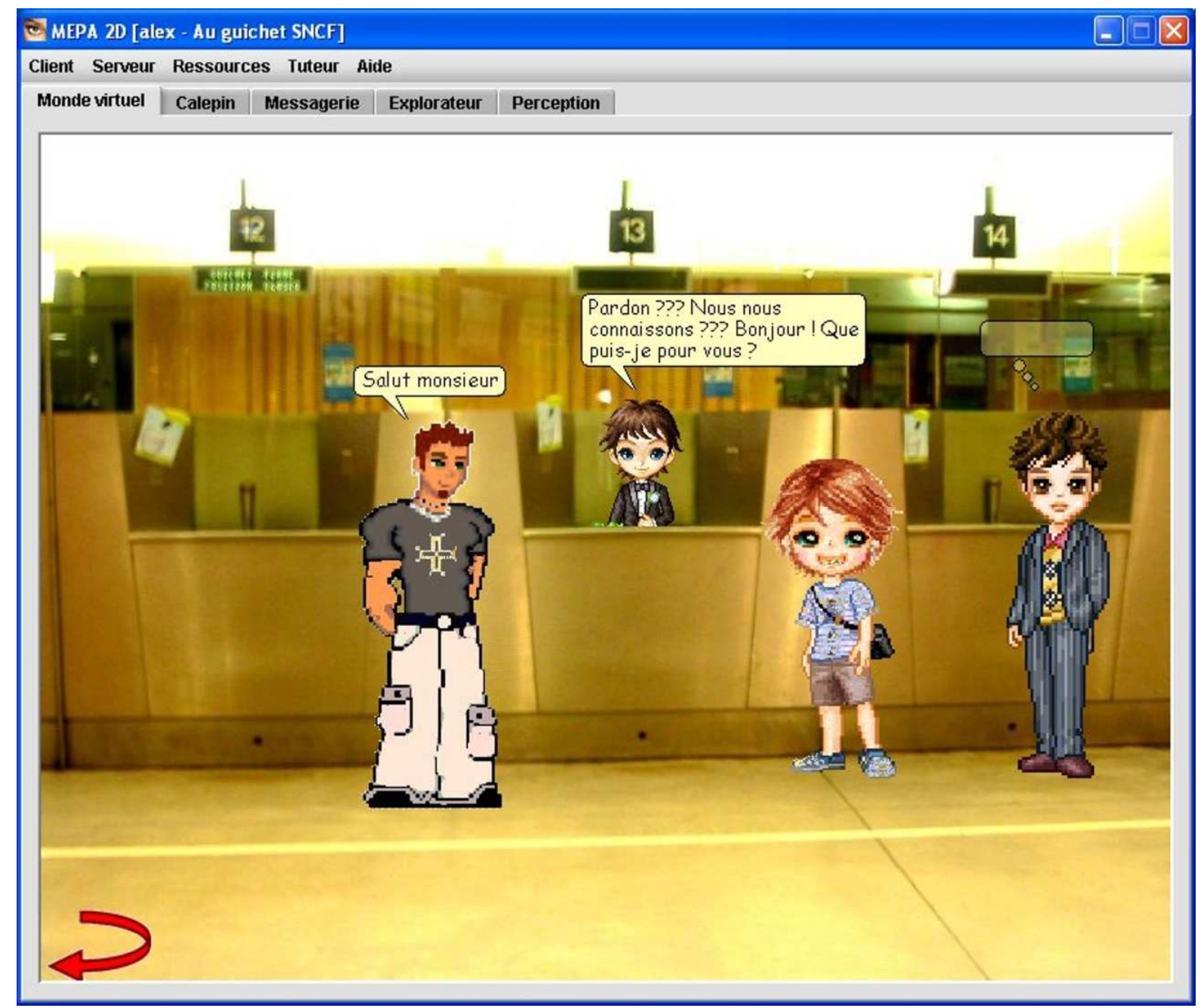

Figure 8 • Scène du guichet SNCF (scène d'entraînement)

Dans les scènes d'évaluation le menu "bulle automatique" n'est plus accessible. Pour obtenir des indices, les apprenants sont obligés de répondre aux questions des agents en engageant leurs propres moyens, en profitant des observations antérieures et en consultant les aides disponibles. L'évaluation s'effectue de manière pragmatique. Face à une multitude d'informations, l'apprenant est obligé de procéder à une sélection pertinente selon ses objectifs. La rétention des renseignements et leur reproduction lui permettront d'estimer ses performances effectives. Son jugement sera alors confirmé/infirmé par l'action déclenchée par le système (indication supplémentaire, résultat immédiat, etc.). La régulation s'accomplit au moyen des interventions des agents logiciels et des tuteurs humains.

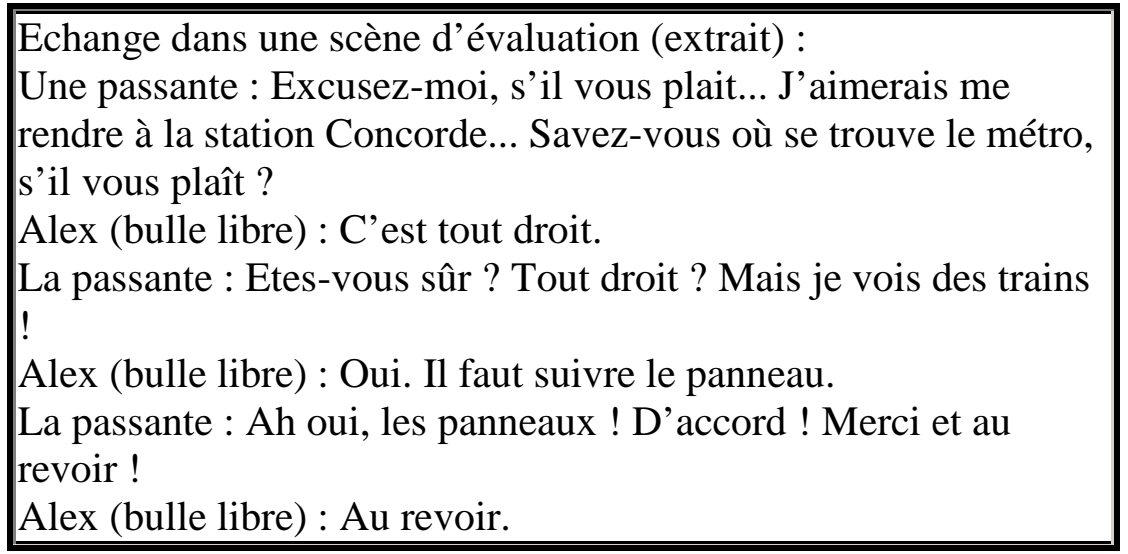




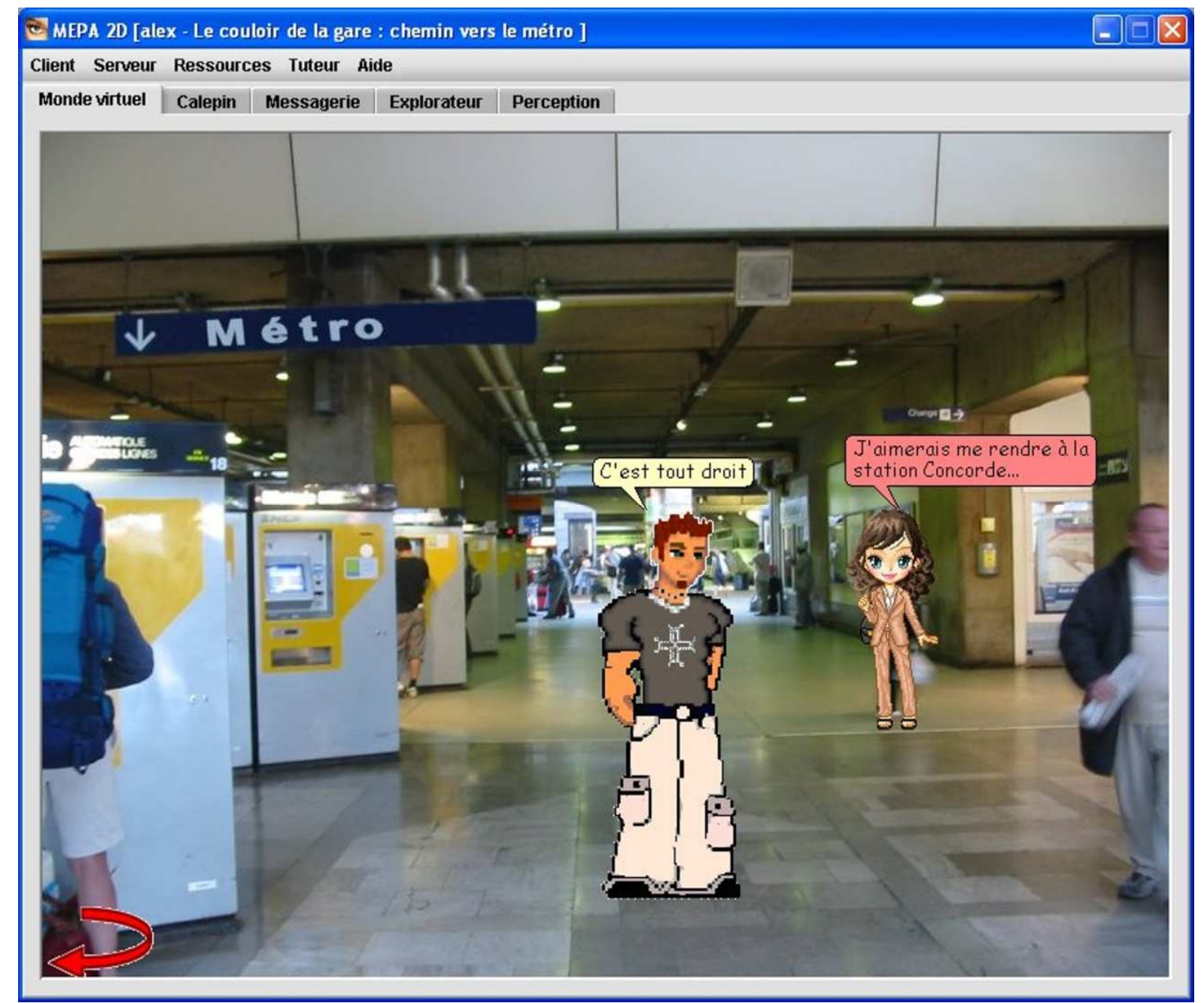

Figure $9 \cdot$ Scène du couloir vers le métro (scène d'évaluation)

\section{Mises à l'épreuve du dispositif}

L'élaboration du prototype s'inscrit dans une démarche itérative et participative. Dans cet esprit, nous avons engagé une série de tests ayant pour but de valider l'utilisabilité de l'environnement, et de recueillir des observations sur la mobilisation des co-constructions argumentées, l'ajustement de la compréhension de la réalité cible, l'aide à l'intercompréhension entre les non-natifs de L1 différentes, le maintien d'une conception partagée du problème et l'élaboration des stratégies de communication. A l'heure actuelle, il est encore trop tôt pour nous prononcer sur l'impact de l'activité sur le processus d'apprentissage. Par contre, un premier examen des interactions nous permet de supposer une incidence du sentiment de réalisme sur les phénomènes cités ci-dessus. Si des analyses plus poussées sont nécessaires pour valider cette hypothèse, d'autres analyses portant sur la morphosyntaxe des échanges sont en cours (Luzzati et al., 2006).

\subsection{Conditions d'observation}

Pour assurer de bonnes conditions d'observation, nous avons décidé de limiter le nombre de participants à 4 par session. Les connexions ont eu lieu aussi bien dans des établissements scolaires (lycées, universités) que dans un cadre privé (connexions des participants depuis leur domicile). Les sessions ont été reparties en 16 séances de $2 \mathrm{~h} 30$ environ, impliquant 6 groupes de 4 apprenants provenant de 5 pays différents (Allemagne, Espagne, Pologne, Thaïlande, Turquie). Au total, les tests ont impliqué 26 apprenants de 17- 
27 ans, de niveau A2-B2 (Conseil de l'Europe, 2001) ainsi que 7 tuteurs (dont 2 concepteurs). Les groupes étaient encadrés si possible par un enseignant en présentiel et par deux tuteurs à distance, ces derniers intervenant sous l'apparence d'avatars. Nous n'avons pu organiser pour l'instant qu'une seule série de sessions croisées impliquant une Allemande, une Polonaise et deux Turques, chacune connectée depuis son pays d'origine. La configuration envisageait aussi un panachage avec des apprenants de niveaux de langue légèrement différents afin d'observer les phénomènes de tutorat entre pairs. En effet, la visualisation du cadre d'intervention partagé par l'ensemble des participants devait a priori faciliter le phénomène d'entraide. Les connexions ont été précédées de présentations de vidéos introductives afin d'amener les participants à émettre des hypothèses en rapport avec le canevas. Plusieurs sessions ont été indispensables pour le parcours complet (3 à 4 sessions). Chaque étape de l'activité a été finalisée par des mises en commun soit en présentiel (par l'enseignant sur place) soit directement dans le monde virtuel (par les tuteurs en ligne). Les différentes sessions ont été suivies de questionnaires d'appréciation destinés à l'ensemble des participants et d'un débat en présentiel. Les impressions des participants ont également pu être partagées sur un forum de discussion.

Ajoutons que les séances de connexion ont produit de grandes quantités de données : les fichiers de traces contiennent plus de $\mathbf{4 4 2 0 0}$ événements dont 11070 prises de parole tout acteur et tout moyen de communication confondus (dans les scènes ou dans la messagerie). Enfin, on comptabilise 2321 prises de parole si on se limite aux seuls apprenants interagissant dans les scènes. Les pourcentages qui suivent se rapportent à ce nombre d'interventions. La forme de ces traces est donnée ci-dessous

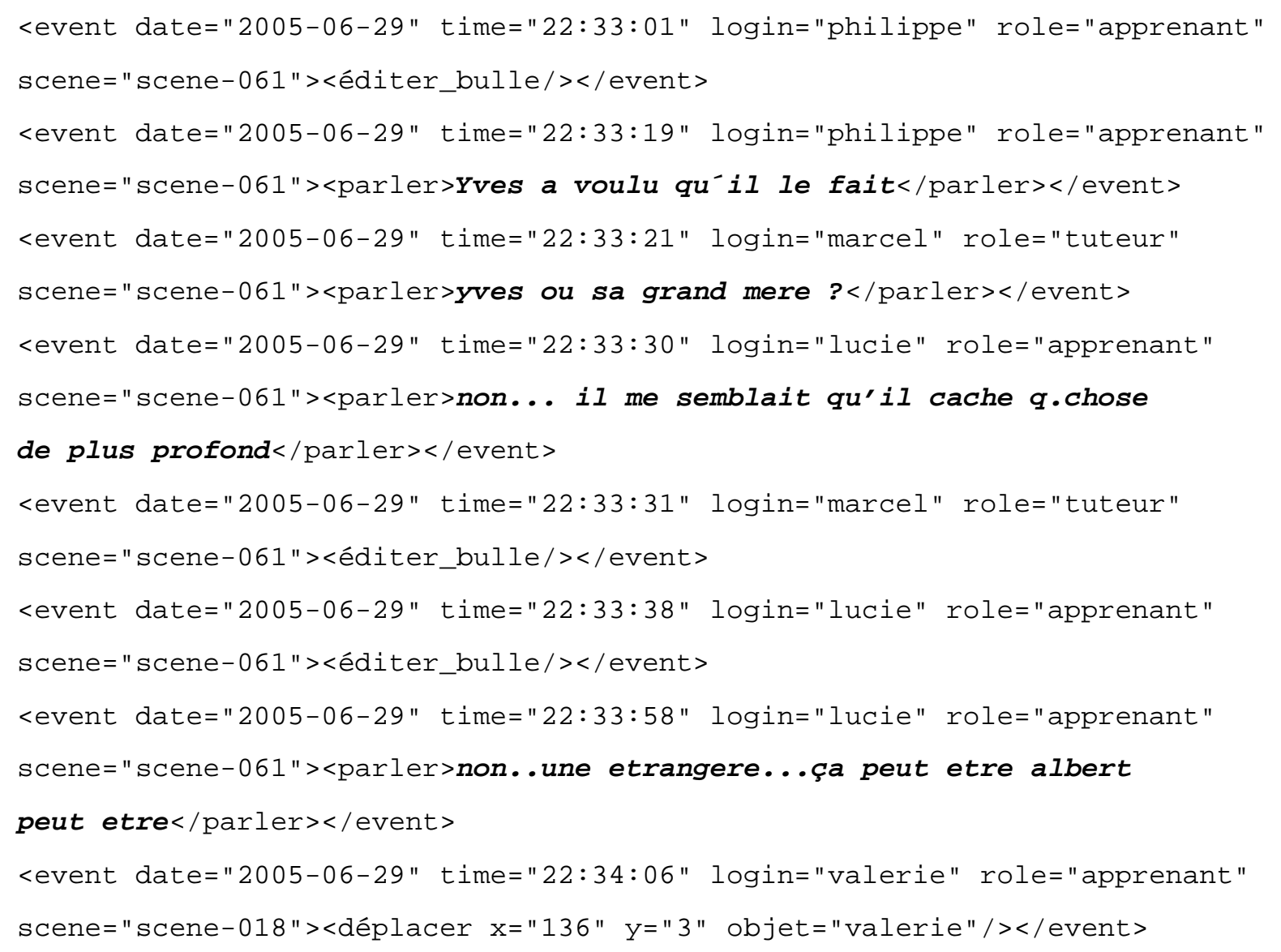

Tableau 3 • Extrait d'un fichier XML de traces

\subsection{Considérations sur les possibilités d'interaction}

Pour interagir dans MEPA-2D les apprenants peuvent recourir à la messagerie instantanée ou bien échanger directement dans le monde virtuel de manière libre ou assistée (actes de parole). Sans aucune 
surprise, l'activité langagière a été très importante. La communication libre (sans passer par le menu "bulle automatique" ) inter-apprenants étant amplement majoritaire. Cette tendance est également confirmée quant à la communication via messagerie instantanée. En ce qui concerne ce dernier mode de communication, nous remarquons certaines évolutions aussi bien quantitatives que qualitatives. Tout d'abord, plus le temps de connexion est important, plus il y a d'échanges entre les apprenants et moins il y a d'échanges entre les tuteurs et les apprenants. La fréquence du recours à ce mode de communication et le nombre de participants impliqués dans l'échange augmentent également. Nous constatons un réajustement du destinataire du message, la présence des citations (à l'aide du copier-coller), ainsi qu'une franche demande de précision (noms propres, déictiques indiciels, descriptions détaillées, etc.) réalisée grâce à la possibilité de se référer à un cadre partagé par l'ensemble des intervenants. Comme pour toute communication médiatisée par ordinateur, il s'agit d'un "écrit oralisé" dont la forme tente de combler l'absence du contexte paralinguistique de la communication face-à-face. Fréquents sont donc les smileys (mimiques faciales), la ponctuation expressive (redoublement du point d'exclamation), l'alternance des majuscules et des minuscules (fonction d'emphase et de focalisation) ou encore l'étirement graphique (ouiiiii). Ces observations coïncident avec les conclusions de nombreux auteurs (Marcoccia, 2004). Toutefois, nous avons également remarqué que pour compenser certaines insuffisances linguistiques, les apprenants recourent à quelques formes iconiques inhabituelles, probablement propres à la communication en L2 dans ce type d'environnement (par exemple des flèches pour désigner directement un objet dans la scène du monde virtuel ou pour indiquer la direction : nous allons >>). Compte tenu des spécificités de ce type de communication, ce procédé peut être qualifié de stratégie compensatoire d'après la typologie de (Oxford, 1990).

L'opportunité d'interagir avec des apprenants d'autres pays a été ressentie d'un commun accord comme fortement motivante grâce à l'authenticité des enjeux communicatifs. En effet, nous n'avons noté que très peu de recours à la langue maternelle durant les connexions croisées alors que cette pratique s'avère plutôt fréquente lorsque les apprenants sont de même origine sociolinguistique. La possibilité d'interagir avec des natifs, fort appréciée, place l'activité dans la perspective co-actionnelle-co-culturelle (Puren, 2001). Les apprenants issus des classes nombreuses, où la prise de parole effective n'est pas toujours garantie, ont considéré le dispositif comme un excellent moyen d'entraînement. En ce qui concerne les échanges avec les agents, les intervenants les jugent suffisants. La majorité des intervenants estiment que les informations dispensées par ces personnages sont importantes notamment pour le déroulement du canevas et donc pour le succès de la mission ludique confiée aux participants. Toutefois, en examinant les traces informatiques, nous avons remarqué un certain nombre de situations où les tuteurs prennent la parole à la place d'un agent logiciel. La plupart de ce type d'interventions (82\%) sont brèves (1 à 4 tours de parole) et ont pour but de réguler les actions des apprenants face aux difficultés organisationnelles ou de relancer les apprenants immobiles dans une scène. 13\% des interventions des tuteurs à travers un agent logiciel concernent les prises de parole conviviales, l'amplification de la personnalisation des réponses des agents ou encore une aide technique (5 à 9 tours de parole). Enfin, 4\% des animations ont lieu pour palier l'absence de réaction de l'agent. Il s'agit des échanges qui n'ont pas pu être prévus par les concepteurs comme les hypothèses émises par les participants. La complexité de ce type d'échange explique leur volume (15 à 26 tours de parole). Au-delà des sujets difficilement prévisibles a priori, les problèmes sont majoritairement causés par les formes de l'écrit oralisé (décrites précédemment), les formes agrammaticales, fragmentaires (écriture consonantique), l'écriture phonétique ou encore les fautes de frappe. Ainsi, il devient urgent d'engager une réflexion sur les performances des agents artificiels dialoguant dans ce type d'environnement.

\subsection{Considérations sur l'impact du sentiment de réalisme}

Une première observation des traces de l'activité nous permet de constater une certaine attention portée aux représentations symboliques des participants. Tout d'abord, les apprenants consultent régulièrement les avatars, non seulement ceux évoquant les autres participants mais également le leur. Ce comportement 
pourrait exprimer une sorte d'appropriation de sa propre représentation symbolique (une sorte d'apprivoisement de son double). De plus, au cours des communications les apprenants orientent systématiquement leur personnage vers leurs interlocuteurs respectifs. Selon le rôle discursif de l'apprenant (locuteur/co-locuteur), cette manipulation peut avoir lieu à deux moments de la communication : avant la prise de parole afin d'annoncer l'intention de communiquer (locuteur) ou bien juste après la prise de parole pour signaler la réception du message (co-locuteur). Cette manipulation de l'avatar semble correspondre, dans une version réduite, à la fonction phatique de la communication faceà-face. Le choix de la forme humanoïde de l'avatar facilement manipulable peut jouer un rôle important pour le potentiel d'identification et la reproduction des dimensions relationnelles de la communication. Aussi, même si les déplacements des participants dans une scène sont libres, la trajectoire de leurs mouvements correspond à celle des circonstances réelles (par exemple longer une rue, un couloir). La reproduction du réalisme, y compris celui des circonstances de la communication, semble affecter les apprenants et avoir des incidences sur la forme de leurs échanges.

Nous avons également observé la fréquence et la manière dont les apprenants explorent les fonds photographiques. L'exploration de l'environnement est plus intense au début de l'activité et elle diminue avec le temps de connexion. Plutôt systématique dans les premières scènes, elle s'organise par la suite en devenant plus intense notamment lorsque le paysage socioculturel change ou bien dans les scènes d'interaction avec les agents logiciels (les éléments évoqués par l'agent peuvent être consultés dans la scène ce qui permet l'accès immédiat au vocabulaire et la vérification des significations opérées). La possibilité de consulter les fonds des scènes contribue aussi à l'efficacité collective des intervenants et au phénomène d'entraide. Ainsi, certains comportements stratégiques de localisation et de guidage ont pu être mis au point à l'aide de la fonction d'exploration et d'observation du cadre socioculturel : informer les camarades virtuellement distants de leur position dans le monde virtuel, les guider, indiquer/vérifier le chemin ou même décrire une manipulation à effectuer. Nous notons que ce phénomène a eu des incidences sur la qualité de la communication entre les apprenants exigeant désormais plus de précisons.

Enfin, les réponses des participants nous permettent de remarquer un enthousiasme unanime pour les ambiances récréées dans les scènes (fonds, bruitages, commentaires sonores) contribuant fortement au sentiment de réalisme. Les apprenants se déclarent suffisamment impliqués dans l'activité pour se mettre dans la peau du personnage. Par conséquent, ils croient pouvoir changer le cours de l'histoire, ce qui les rend actifs et maintient leur motivation. Les participants ont noté une meilleure mémorisation et appropriation du matériau linguistique dues à sa présentation en situation, suivie d'une manipulation immédiate. L'activation du vocabulaire passif est souvent citée comme un des avantages de la mise en situation ancrée aussi bien au niveau linguistique que socioculturel.

\section{Conclusion}

L'objectif de cet article est moins de valider des hypothèses que de soumettre une approche et un environnement logiciel la supportant dédiée à la pratique collective et à distance d'une langue étrangère, ainsi que d'engager une réflexion sur le potentiel didactique d'environnements de ce type. A partir d'un bref état de l'art, nous avons dégagé un ensemble de critères qui permettent de caractériser les platesformes dédiées à la pratique communicative d'une langue étrangère, ou utilisées comme tel dans le cadre d'activités de type SGR (Simulation Globale en Réseau). Nous avons évoqué le problème de la complexité des mondes virtuels et donc du coût de leur conception. Enfin, nous avons décrit MEPA-2D, une nouvelle plate-forme de type SGR, que nous avons prototypée et mise à l'essai dans des contextes et avec des publics variés. En favorisant la collaboration au sein d'un groupe d'apprenants encadrés par un réseau de tuteurs, MEPA-2D tente d'articuler les principes du socioconstructivisme, de l'étayage Brunerien et de l'apprentissage collaboratif. Par rapport aux autres environnements inspirés de la SG, MEPA-2D semble apporter certains avantages comme le coût minimum, la manipulation (réédition) et la contextualisation des actes de parole ainsi que la possibilité d'adaptation des contenus par l'enseignant. 
Le dispositif réussit également à répondre à la majorité des critères retenus bien que quelques concessions semblent inévitables. Compte tenu de ces compromis, peut-on toujours parler de simulation globale ? La dimension globale de l'activité préconisée par Francis Debyser se traduit avant tout par l'étendue du contexte permettant de fédérer toutes les activités d'apprentissage autour d'un thème et par la coconstruction successive d'un monde vécu (composition des identités fictives et celle d'un lieu-thème). Si, grâce au recours à la représentation graphique amplifiant le degré de réalisme, le dispositif respecte systématiquement le premier principe (contexte), la créativité des apprenants peut être toujours considérée comme limitée. Les technologies actuelles ne permettent pas de co-construire facilement un monde dont l'évolution serait instantanément perceptible à l'écran. Néanmoins, MEPA-2D le permet de manière différée et s'efforce de multiplier les opportunités de la créativité langagière. De plus, les apprenants construisent leurs identités fictives et ont une certaine liberté de parcours qui leur donne l'impression d'influencer le canevas de la simulation. Ainsi, la "globalité" peut être également considérée comme le degré de l'investissement de l'apprenant dans son activité. Par ailleurs, les simulations globales naissent en réaction aux insuffisances des simulations simples pratiquées pour développer la compétence de communication (faible ancrage socioculturel, manque de réels enjeux communicatifs, simple reconstitution des dialogues prédéfinis, etc.). Il en est de même dans MEPA-2D. La qualité de la mise en situation, la richesse du contexte, la dynamique de l'action et la possibilité de la personnaliser, ne nous permettent pas de la considérer comme "simple". Pour ces différentes raisons nous qualifions cette activité de globale, même si cette globalité ne se traduit pas toujours de la même manière que celle préconisée par Francis Debyser.

Les premiers essais de MEPA-2D se sont révélés extrêmement intéressants, tant du point de vue de l'usage de l'environnement (par les apprenants et les tuteurs), que du point de vue des pistes de recherches qui en découlent. La pratique de l'activité de tutorat a mis en valeur la nécessité de développer une instrumentation spécifique à ce type d'environnement, tant au niveau de la perception de l'activité des apprenants, que de l'intervention, ou encore de la gestion de l'activité tutorale elle-même. Ces trois niveaux sont ceux du modèle SAAD (Després, 2003) de suivi pédagogique synchrone à partir duquel nous nous proposons de travailler.

Malgré certaines difficultés rencontrées durant les premières expérimentations (lenteur de certaines connexions, bugs résiduels ou déroulement des tests sur plusieurs jours entravant quelque peu la dynamique du jeu), de nombreux apprenants et enseignants se sont montrés intéressés pour participer à d'autres tests. Cet enthousiasme des premiers utilisateurs, qui de plus étaient non captifs (participation sur la base du volontariat, pas d'évaluation, simple engagement moral), nous permet de croire à un avenir prometteur des environnements de SGR en général et de ce type de dispositif en particulier.

\section{BIBLIOGRAPHIE}

ARNAUD M., SERDIDI M. (2003). Conditions favorables à la construction des connaissances avec les jeux de rôles sur Internet, In Missonnier S. \& Lisandre H. (Eds.). Le Virtuel : la présence de l'absent, Paris, EDK.

BELZ J.A., KINGINGER C. (2002). Cross-linguistic development of address form use in telecollaborative language learning: two case studies, Revue Canadienne des Langues Vivantes (59), $\mathrm{p}$. 189-214.

BRUN C., PARMENTIER T., SANDOR A., SEGOND F. (dir) (2002). Les outils de TAL au service de la e-formation en langues, Multilinguisme et traitement de l'information, Hermès Science Publications, $\mathrm{p}$. 223-250.

CHANIER T. (2002). Créer des communautés d'apprentissage à distance, Les dossiers de l'Ingénierie Educative (36), p. 56-59. 
CONSEIL DE L'EUROPE (2001). Cadre européen commun de référence pour les langues : apprendre, enseigner, évaluer, Didier, Paris.

DEAUDELIN C., DUBE C. (2003). Collaboration en face à face et à distance. Interactions d'élèves du primaire axées sur la négociation, In Deaudelin C. et Nault T. (dir) Collaborer pour apprendre et faire apprendre. La place des outils technologiques, Presses de l'Université du Québec, p. 135-154.

DEBYSER F. (1974). Simulations et réalité dans la classe de langue, Le Français dans le Monde (104), p. 6-10.

DEBYSER F. (1991). Les simulations globales, Education et Pédagogies (10), CIEP.

DESPRES C. (2003). Synchronous Tutoring in Distance Learning, Artificial Intelligence in Education (AIED), 20-24 juillet 2003, Sydney (Australie), p. 271-278.

DE CARLO M. (1998). L’interculturel, Clé International, Paris.

DEWAELE J.-M. (2002). Variation, chaos et système en interlangue française, In Dewaele J.-M., Mougeon R. (Eds.). Appropriation de la variation en français langue étrangère, AILE (17), p. 143-167.

DILLENBOURG P., BAKER M. (1996). Negotiation spaces in human-computer collaborative learning, Second International Conference on Design of Cooperative Systems, Proceedings of COOP, p. 187-206.

HENRI F., LUNDGREN-CAYROL K. (2001) Apprentissage collaboratif à distance. Pour comprendre et concevoir les environnements d'apprentissage virtuels, Presses de l'Université du Québec.

JOHNSON W.L., MARSELLA S., MOTE N., VILHJALMSSON H., NARAYANAN S., CHOI S. (2004). Tactical Language Training System Supporting the Rapid Acquisition of Foreign Language and Cultural Skills, Proceedings of InSTIL/ICALL2004 - NLP and Speech Technologies in Advanced Language Learning Systems, 17-19 June 2004, Venice (Italy).

JOHNSON W.L., VILHJALMSSON H., MARSELLA S. (2005). Serious games for language learning: How much game, how much AI ?, Artificial Intelligence in Education (AIED).

JONES J.G. (2004). 3D On-line Distributed Learning Environments: An Old Concept with a New Twist, Proceedings of the Society for Information Technology and Teacher Education, Atlanta, GA, p. 507-512.

JONES J.G., MORALES C., KNEZEK G. (2004). 3D Graphical Multi-User Online Learning Environments for Internet-based Distributed Learning: First Year Results, Proceedings of the National Educational Computing Conference, New Orleans, LA.

LUZZATI D., LEHUEN J., KITLINSKA S. (2006). Quelques pratiques langagières dans MEPA-2D, un dispositif de simulation globale en ligne pour la pratique du Français-Langue Etrangère, Actes du colloque CMT 2006 : la langue de la communication médiatisée par les technologies de l'information et de la communication, 18-20 mai 2006, Bordeaux (France).

LYSTER R., REBUFFOT J. (2002). Acquisition des pronoms d'allocution en classe de français immersif, In Dewaele J.-M. et Mougeon R. (Eds.). Appropriation de la variation en français langue étrangère, AILE (17), p. 51-72.

MANGENOT F. (1996). Les aides logicielles à l'écriture, In Linguistique, informatique, pédagogie : simulation et modélisation, Paris, CNDP, p. 69-117.

MANGENOT F. (1998). Classification des apports d'Internet à l'apprentissage des langues, Revue ALSIC, Vol. 1 (2).

MARCOCCIA M. (2004). La communication écrite médiatisée par ordinateur : faire du face à face avec de l'écrit, Journée d'étude ATALA : Le traitement automatique des nouvelles formes de communication 
écrite.

MOUGEON R., NADASDI, T., REHNER, K. (2002). État de la recherche sur l'appropriation de la variation par les apprenants avancés du FL2 ou FLE, Dewaele J.-M., Mougeon R. (Eds.). Appropriation de la variation en français langue étrangère, AILE (17), p. 7-50.

OXFORD R. (1990). A new Taxonomy of Second Language Learning Strategies, Washington, D.C., ERIC Clearing House on Languages and Linguistics.

PAPERT S. (1980). Mindstorms: Children, Computers, and Powerful Ideas, Basic Books, New-York.

PUREN C. (2001). Les nouvelles technologies face aux nouvelles options didactiques, Actes du Colloque apprendre les langues européennes avec les nouvelles technologies, Paris, Goethe-Institut.

REFFAY C., CHANIER T., NICOLET J. (2002). Produire ensemble pour apprendre : expérience d'une simulation globale en ligne, Actes du colloque Apprendre avec l'ordinateur à l'école, Bordeaux (France).

SEGOND F., PARMENTIER T., STOCK R., ROSNER R., USTERAN-MUELA, M. (2005). Situational language training for hotel receptionists, Proceedings of the 2nd Workshop on Building Educational Applications Using NLP, p. 85-92.

YAICHE F. (1996). Les simulations globales, mode d'emploi, Paris, Hachette.

\section{SITOGRAPHIE (derniers accès le 06/10/06)}

http://www.exills.com

http://home.sandiego.edu/ mmagnin/hotel.html

http://lucke.univ-lemans.fr/mepa

http://www.umsl.edu/ moosproj/moofrancais.html

http://www.web3d-fr.com/articles/blaxxun/Xerox/article.php

http://www.tcd.ie/CLCS/assistants/kschwien/Publications/CALLMOOtalk.htm

http://www.isi.edu/isd/carte/proj_tactlang

http://web.syr.edu/ lmturbee/itechtm.html

http://www.prologue.qc.ca 


\section{- A propos des auteurs}

Jérôme LEHUEN est maître de conférences en Informatique à l'Université du Maine. Il s'intéresse aux EIAH pour l'apprentissage d'une langue dans l'interaction et au dialogue personne-machine en langue naturelle.

Adresse : LIUM, Université du Maine, Avenue Laënnec, 72085 LE MANS CEDEX 9

Courriel : $\underline{\text { Jerome.Lehuen@ lium.univ-lemans.fr }}$

Toile : http://www-lium.univ-lemans.fr/ lehuen

Sylwia KITLINSKA est doctorante en Sciences du Langage à l'Université du Maine. Sa spécialité est la didactique du FLE et elle s'intéresse à la conception et à l'usage d'environnements de simulation pour l'apprentissage du FLE.

Adresse : LIUM, Université du Maine, Avenue Laënnec, 72085 LE MANS CEDEX 9

Courriel : Sylwia.Kitlinska@lium.univ-lemans.fr

(1) Situation où la langue étrangère enseignée n'est pas utilisée de manière significative en dehors de l'espace classe. Concernant le FLE, il s'agit des situations d'apprentissage où les non-natifs apprennent la langue en dehors de la France ou de tout pays francophone (le français dans ces pays a un autre statut que celui d'une langue étrangère).

(2) Le terme "L2" est employé dans cet article par opposition à "L1" comme synonyme de la langue cible sans faire référence à la langue seconde.

(3) Nous la qualifions d'artificielle puisqu'elle s'effectue de manière ponctuelle, en milieu scolaire, le plus souvent hétéroglotte.

${ }^{(4)}$ Multiuser Object Oriented (environnement) / Multi User Dungeon (MUD) Object Oriented.

(5) Xerox Research Centre Europe.

(6) Les agents logiciels de MEPA-2D ne sont pas décrits dans cet article. Il s'agit d'agents conversationnels programmables (règles de dialogue/comportement) capables de produire un énoncé et d'effectuer des actions en fonction d'un contexte interactionnel verbal et non-verbal (déposes d'objets, actions, attitudes, etc.). Cet aspect fait l'objet de travaux en cours.

\section{Référence de l’article :}

Jérôme LEHUEN, Sylwia KITLINSKA, Simulation Globale en Réseau pour le FLE ? La Plate-forme Informatique MEPA-2D, Revue STICEF, Volume 13, 2006, ISSN : 1764-7223, mis en ligne le 28/11/2006, http://sticef.org

(c) Revue Sciences et Technologies de l’'Information et de la Communication pour l'Éducation et la Formation, 2006 